\title{
Decomposition of Multiple Coverings into More Parts
}

\author{
Greg Aloupis · Jean Cardinal • Sébastien Collette • \\ Stefan Langerman • David Orden • Pedro Ramos
}

Received: 6 March 2009 / Revised: 17 December 2009 / Accepted: 18 December 2009 /

Published online: 8 January 2010

(C) Springer Science+Business Media, LLC 2010

\begin{abstract}
We prove that for every centrally symmetric convex polygon $Q$, there exists a constant $\alpha$ such that any locally finite $\alpha k$-fold covering of the plane by translates of $Q$ can be decomposed into $k$ coverings. This improves on a quadratic upper bound proved by Pach and Tóth. The question is motivated by a sensor network problem, in which a region has to be monitored by sensors with limited battery life.
\end{abstract}

Keywords Cover decomposition · Geometric hypergraphs · Centrally symmetric polygons

Part of this work was done while the second and fourth authors where visiting Universidad de Alcalá, under the program Giner de los Ríos.

G. Aloupis, J. Cardinal, S. Collete and S. Langerman are supported by the Communauté Française de Belgique.

D. Orden and P. Ramos are supported by grant MTM2008-04699-C03-02.

S. Collette is Chargé de Recherches du FRS-FNRS.

S. Langerman is Maître de Recherches du FRS-FNRS.

G. Aloupis · J. Cardinal · S. Collette $(\varangle) \cdot$ S. Langerman

Université Libre de Bruxelles (ULB), CP212, Bld. du Triomphe, 1050 Bruxelles, Belgium

e-mail: secollet@ulb.ac.be

G. Aloupis

e-mail: greg.aloupis@ulb.ac.be

J. Cardinal

e-mail: jcardin@ulb.ac.be

S. Langerman

e-mail: slanger@ulb.ac.be

D. Orden · P. Ramos

Universidad de Alcalá, Alcalá, Spain

D. Orden

e-mail: david.orden@uah.es

P. Ramos

e-mail: pedro.ramos@uah.es 


\section{Introduction}

A collection of subsets of the plane forms an $f$-fold covering if any point in the plane is covered by at least $f$ subsets. We consider the following problem (see Fig. 1):

Given a convex planar body $Q$, does there exist a function $f(Q, k)$ such that any $f(Q, k)$-fold covering of the plane by translates of $Q$ can be decomposed into $k$ disjoint (1-fold) coverings?

This problem, first raised by Pach in 1980 (see [10] and references therein), is a classical question in discrete geometry (see, e.g., $[9,11,16])$ and remains largely open. In fact it is not even known whether there exists a constant $c$ such that any $c$-fold covering can be decomposed into two coverings. A survey of the literature can be found in the book of Brass, Moser, and Pach [4]. Note that there is no such function in general; for certain concave polygons, this was first shown in [12], and it was extended to a larger class of concave polygons by Pálvölgyi [14].

In an unpublished manuscript, Mani and Pach [9] claim that 33-fold coverings by open unit disks can be decomposed into two coverings. ${ }^{1}$ Tardos and Tóth recently proved that any 43-fold covering by translates of an open triangle can be decomposed into two coverings; they proved an exponential upper bound on $f(Q, k)$ for open triangles [16].

For the case of translates of an open centrally symmetric convex polygon, the problem proved to be challenging. The existence of a function $f(Q, k)$ was conjectured in 1980 [10], and a few years later, resolved positively [11] by Pach, showing that $f(Q, k)$ is at most exponential in $k$. Only twenty years later it was shown that $f(Q, k)$ is at most quadratic.

Theorem 1 (Pach and Tóth [13]) Given a centrally symmetric open convex polygon $Q$, there exists a constant $\alpha_{Q}$ such that every $\alpha_{Q} k^{2}$-fold covering of the plane by translates of $Q$ can be decomposed into $k$ coverings.

Fig. 1 A 3-fold covering of a rectangle by hexagons that can be decomposed into three coverings
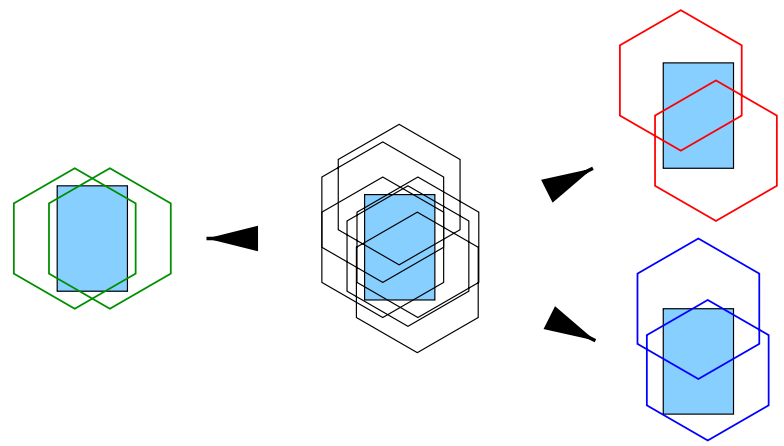

\footnotetext{
${ }^{1}$ In the November 2009 issue of the Communications of the ACM, Perter Winkler poses this as an open problem. He conjectures that 4-fold coverings with unit disks are decomposable.
} 
In addition to the above, a lower bound of $\lfloor 4 k / 3\rfloor-1$ was also given in [13].

The main result in this paper is an improvement of the bound in Theorem 1, from $\alpha_{Q} k^{2}$ to $\alpha_{Q} k$; thus, the upper and lower bounds now asymptotically match.

Note that for translates of an arbitrary open convex polygon, Pálvölgyi and Tóth recently proved that there exists a constant $c$ such that $c$-fold coverings can be decomposed into two coverings [15]. This leads to a high-degree polynomial upper bound on the function $f(Q, k)$ for translates of an arbitrary open convex polygon.

As mentioned above, previous results hold for open bodies and for arbitrary coverings. By requiring that the covering is locally finite, the results hold for both open and closed bodies. This observation was made in previous papers and was first discussed in [16]; the problem is still open for unrestricted coverings by closed convex bodies.

In what follows, we require that the covering is locally finite, and we solve the problem for translates of a centrally symmetric closed polygon. Our results do not rely on the closeness of polygons (we can slightly perturb the input to avoid the open/closed issue), and therefore our results apply, similar to most other results in the field, to unrestricted coverings by open centrally symmetric polygons and to locally finite coverings by either open or closed centrally symmetric polygons.

Related Work Coverings with other families of convex shapes have also been studied. For instance, indecomposable multiple coverings of the plane by strips and concave quadrilaterals were given by Pach, Tardos, and Tóth [12] and for large classes of concave polygons by Pálvölgyi [14]. The problem for arbitrary (open) disks remains open, although a negative result for the dual problem was proved in [12]: for any $k$, there exists a point set such that for any 2-coloring of this set, an open disk containing $k$ points of the same color can be found. Set-theoretic investigations of infinite-fold coverings can be found in [6].

Note that decompositions of coverings can be seen as colorings of geometric hypergraphs. In these hypergraphs, vertices are the convex bodies in the covering, and every point in the plane corresponds to a hyperedge, defined as the set of bodies containing that point. The assignment of colors to the vertices of this graph, such that every hyperedge contains all $k$ colors, yields a suitable decomposition. A recent study of such problems and of their duals, including colorings of hypergraphs induced by halfspaces, halfplanes, disks, and pseudo-disks, is presented in [3]. ${ }^{2}$

Similar definitions of proper colorings of geometric hypergraphs have been studied, such as conflict-free colorings [7]. Here the problem is to find a coloring such that every hyperedge contains at least one vertex with a unique color. Variants of this notion have also been analyzed, e.g., $k$-fault-tolerant conflict-free colorings where the conflict-free property must be true even if we were to remove any $k$ vertices in a hyperedge [1]. $k$-conflict-free colorings [2] require $k$ vertices with unique colors in every hyperedge.

Applications to Sensor Networks Consider a planar region monitored by sensors. Each sensor is represented as a point, which is said to monitor every other point contained in a polygonal region around it. Sensors can be on for a fixed amount of

\footnotetext{
${ }^{2}$ Using the notation of [3], the main result of this paper is that $p_{\overline{\mathcal{T}}}(k)=O(k)$.
} 
time and can be switched on at any chosen time. Such models of limited-battery sensors have been studied in other contexts [5]. Our results imply that a region can be monitored for $k$ units of time, provided that every point is covered by at least $\alpha k$ sensors. This involves partitioning the set of sensors into $k$ subsets, each covering the region. Sensors in the $j$ th subset are switched on at time $j$.

Problem Modification We now slightly modify the statement of the problem. Let $Q_{p}$ denote a centrally symmetric polygon $Q$ centered at point $p$. Notice that $Q_{p}$ covers a point $p^{\prime}$ if and only if $Q_{p^{\prime}}$ contains $p$.

The problem involves a set of translates of $Q$ that covers every point of the plane at least $\alpha_{Q} k$ times. This is geometrically equivalent to a point set $S$ such that any translate of $Q$ in the plane contains at least $\alpha_{Q} k$ points of $S$. Note that, of course, $S$ must be infinite, as must be a covering in the original problem.

The decomposition of translates into $k$ covers is equivalent to a coloring of $S$ such that every translate of $Q$ in the plane will contain $k$ colors. We strengthen the problem statement by relaxing the condition that every translate in the plane contains sufficiently many points. That is, we say that if a translate contains enough points, it will contain $k$ colors. This allows us to consider finite point sets as well. We thus prove the following result.

Theorem 2 Given a centrally symmetric convex polygon $Q$, there exists a constant $\alpha_{Q}$ such that for every (locally finite) planar point set $S$ and every $k \in \mathbb{N}, S$ can be $k$-colored so that any translate of $Q$ containing at least $\alpha_{Q} k$ points will also contain at least one point of each color.

After the initial submission of this manuscript, Gibson and Varadarajan [8] extended Theorem 2 by showing that translates of an arbitrary convex polygon can be decomposed into a linear number of coverings. Their methods rely heavily on the results presented in the following sections.

For simplicity of exposition, we assume that no two points in $S$ have the same slope as an edge of $Q$. This assumption can be removed by applying an infinitesimal perturbation to the points. Also, we assume that $S$ is locally finite; every compact region contains a finite number of points.

Overview We start by giving a sketch of the complete proof before going into details. The original problem is transformed as follows.

The problem of coloring a (possibly infinite) point set with respect to translates of a polygon (the strengthened statement presented in Theorem 2 ) is shown to be equivalent to coloring a finite point set with respect to a finite set of wedges determined by $Q$ (see Sect. 2). In other words, the problem is now to $k$-color a set of points such that every wedge containing a sufficient number of points $m$ will also contain $k$ colors. Our goal is to show that $m=O(k)$.

We will restrict to color points inside certain witness wedges which have the property that any wedge containing at least $m$ points will contain a witness. Witnesses will contain at least $r$ points. This is why we will define the level curve which bounds the union of such minimal wedges for a fixed pair of bounding directions (see Sect. 2). 
If the level curves did not intersect, coloring the points would be straightforward. It is the intersections of these curves that make the problem nontrivial and forbid us to restrict to witnesses on level curves only. Since $k$ is small with respect to the point set, intuitively one can imagine that level curves tend not to venture too "deep" into a point set. In other words, a typical wedge will not reach far into the set before collecting $r$ points. In Sect. 3 we define a polygonal region that is deep enough so that the complexity of level curve intersections within the region is manageable. Our construction of this region will be such that we will be able to restrict to considering witness wedges within.

To reduce our problem to circular arc coloring, in Sect. 4 we define a parameterization which maps the set of witness wedges to the boundary of a circle. This is directly tied to a mapping of points in $S$ to circular arcs, i.e., intervals on the boundary of the circle (Sect. 5). Our mapping is such that a position $x$ on the circle belongs to an interval corresponding to point $p \in S$ if and only if the witness wedge represented by $x$ contains $p$. As every witness wedge contains at least $r$ points, every position on the circle belongs to at least $r$ intervals. The key property of the parameterization is that every point in $S$ is mapped to at most two intervals.

Thus, the problem is reduced to $k$-coloring arcs on a $\Theta(k)$-covered circle (with certain geometric constraints for the arcs), so that every position on the circle is covered by at least one interval of each color. In Section 6 we give an algorithm for this circular arc coloring problem.

Note that the reductions and transformations of the problem are constructive; thus our algorithm to color circular arcs yields a simple polynomial algorithm for the original problem.

\section{Reduction to Wedges}

Let $Q$ be a closed, convex, centrally symmetric $2 n$-gon, with vertices $q_{0}, q_{1}, \ldots$, $q_{2 n-1}$ in counterclockwise order. Throughout the paper, indices are taken modulo $2 n$. The set of indices between $i$ and $j$ in counterclockwise order is denoted by $[i, j]$.

We first reduce the problem to coloring a finite set of points with respect to wedges instead of coloring a possibly infinite set with respect to polygons. This idea is also used in $[13,16]$.

We consider a tiling of the plane, with squares of side $\delta$, where $\delta$ is half of the smallest distance between nonconsecutive edges of $Q$. Let $Q^{\prime}$ be a translate of $Q$. By construction, any intersection of $Q^{\prime}$ with a square is the intersection of a square with a wedge with boundary directions parallel to two consecutive edges of $Q$ (see Fig. 2). A wedge bounded by rays parallel to $q_{i} q_{i-1}$ and $q_{i} q_{i+1}$ will be called type $i$, or alternatively an $i$-wedge. The closed $i$-wedge with apex $x$ is denoted by $W_{i}(x)$.

The number of squares that $Q^{\prime}$ intersects is bounded by a constant $c_{Q}$ that only depends on $Q$. Therefore if $Q^{\prime}$ contains at least $\alpha_{Q} k$ points, by the pigeonhole principle $Q^{\prime}$ contains at least $\alpha_{Q} k / c_{Q}$ points within one of the squares.

We will restrict to considering a single square and the $2 n$ wedges defined by $Q$. Hence the problem reduces to coloring (independently) the finite bounded point set $S$ in each square, i.e., we will seek a $k$-coloring of each square such that any $i$-wedge containing at least $\alpha_{Q} k / c_{Q}$ points will contain all $k$ colors. 
Fig. 2 Reduction of the problem with centrally symmetric polygons to wedges in a square

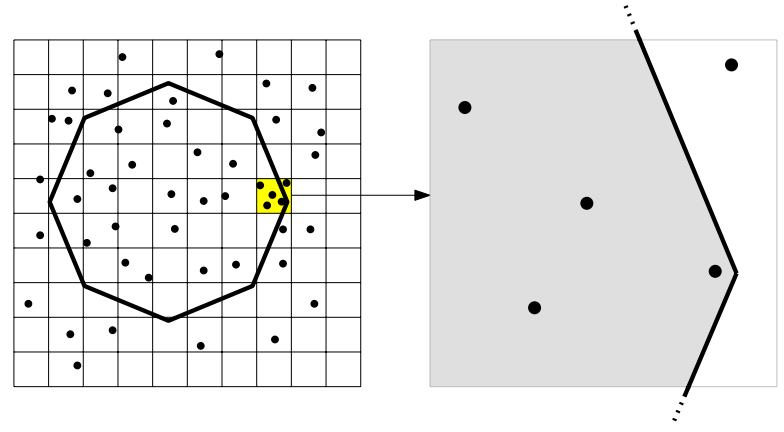

We now define the notion of level curves for wedges. This notion extends the definition of boundary points in [13] and [11], which are the points found on the first level. We associate a curve with each $i$-wedge. Let $\mathcal{W}_{i}^{r}$ be the set of spices of all $i$-wedges containing $r$ points. Formally,

$$
\mathcal{W}_{i}^{r}:=\mathrm{Cl}\left(\left\{x \in \mathbb{R}^{2}:\left|W_{i}(x) \cap S\right|=r\right\}\right),
$$

where $\mathrm{Cl}(\cdot)$ is the closure operator. We define $\mathcal{C}_{i}(r)$ as the boundary of $\mathcal{W}_{i}^{\geq r}:=$ $\bigcup_{j \geq r} \mathcal{W}_{i}^{j}$. Accordingly, the closed region that includes the complement of $\mathcal{W}_{i}^{\geq r}$ will be denoted $\mathcal{W}_{i}^{<r}$ (i.e., the intersection of the two regions is $\mathcal{C}_{i}(r)$ ).

Note that $\mathcal{C}_{i}(r)$ is a monotone staircase polygonal path, with edge directions parallel to those of its corresponding $i$-wedge. Since $S$ is in general position, for any $x \in \mathcal{C}_{i}(r)$ that is not a vertex of $\mathcal{C}_{i}(r), W_{i}(x)$ contains exactly $r$ points. More precisely, we have the following:

Observation 1 For all $x \in \mathcal{C}_{i}(r), W_{i}(x)$ contains either $r$ or $r+1$ points of $S$.

The curves $\mathcal{C}_{i}$ (3) for a square are illustrated in Fig. 3. A key property of $\mathcal{C}_{i}$ is the following:

Observation 2 Any $i$-wedge containing at least $r$ points of $S$ contains an $i$-wedge whose apex belongs to $\mathcal{C}_{i}(r)$.

We conclude that it is sufficient to color points in the union of all $\mathcal{W}_{i}^{<r}$ (in other words, in the union of regions to the "left" of each $\mathcal{C}_{i}(r)$ ). Handling the complexity of the intersections of these curves is the next problem that we deal with.

\section{Restriction to High-Depth Region}

We will show that in order to determine the witness wedges that we must color, it is not necessary to consider complete level curves. At the expense of a constant factor to $f(Q, k)$, we restrict to the portion of the level curves inside a polygon $\mathcal{T}$. Inside this polygon, only few intersections between level curves can occur, which simplifies the coloring task. 
Fig. 3 The curves of $\mathcal{C}_{i}(3)$ when $Q$ is an axis-parallel square

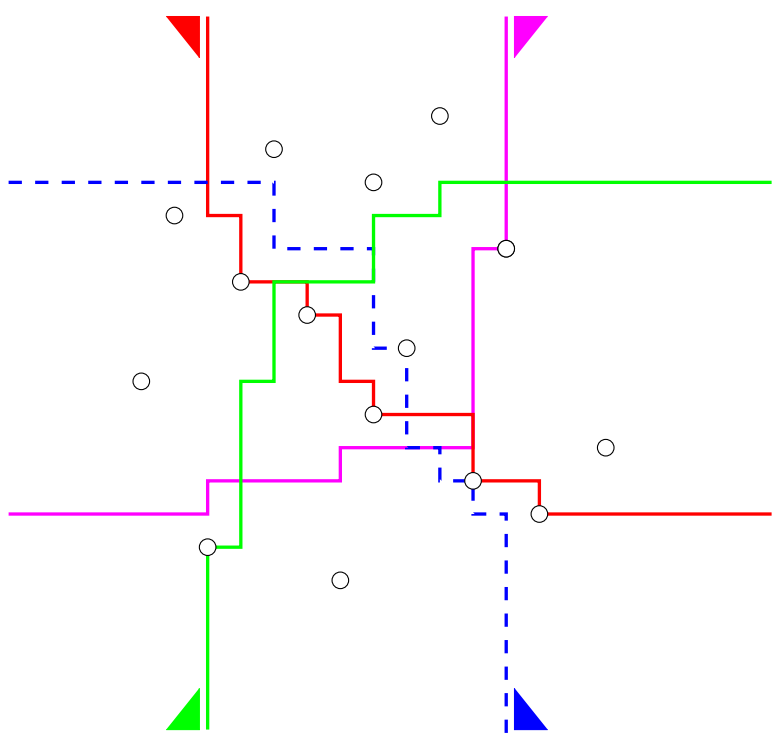

Let $\ell_{i}$ be the oriented line with direction $q_{i} q_{i+1}$ going through a point of $S$ and such that the closed halfplane to its left contains exactly $2 r+3$ points. Let $L_{i}$ be the closed halfplane to the right of $\ell_{i}$. Denote by $\mathcal{T}$ the intersection of the $2 n$ halfplanes defined by $Q$ :

$$
\mathcal{T}:=\bigcap_{i=0}^{2 n-1} L_{i} .
$$

We assume that $\mathcal{T} \neq \emptyset$ : this will be shown true later for the values of $r$ that we will use (by the well-known center point theorem, it is true as long as $2 r+3 \leq|S| / 3$ ). Note that not all lines $\ell_{i}$ appear on the boundary of $\mathcal{T}$ (see Fig. 4(a)).

Lemma 1 For all $i \in[0,2 n-1]$, there is a vertex $v_{i}$ of $\mathcal{T}$ such that $v_{i} \in W_{i}(x)$ for all $x \in \mathcal{T}$.

Proof Let $\hat{\ell}_{i}$ be the oriented line parallel to $\ell_{i}$ that is tangent to $\mathcal{T}$ and such that $\mathcal{T}$ is contained in the closed halfplane to the right of $\hat{\ell}_{i}$. Then for

$$
v_{i}:=\hat{\ell}_{i} \cap \hat{\ell}_{i-1}
$$

the wedge $W_{i+n}\left(v_{i}\right)$ contains $\mathcal{T}$. Therefore, $v_{i} \in W_{i}(x)$ for all $x \in \mathcal{T}$. Note that a vertex of $\mathcal{T}$ may have multiple labels $v_{i}$ (see Fig. 4(a)).

Lemma 2 Let $x$ be a point contained in two wedges $W_{i}(y)$ and $W_{j}(z)$ that contain at most $r$ and $r^{\prime}$ points of $S$, respectively, with $0<(j-i)<n$. Then for all $i^{\prime} \in[i, j-1]$, the oriented line with direction $q_{i^{\prime}} q_{i^{\prime}+1}$ through $x$ has at most $r+r^{\prime}$ points of $S$ strictly to its left. 


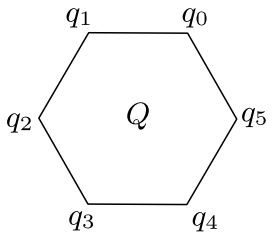

(a) Definition of the points $v_{i}$

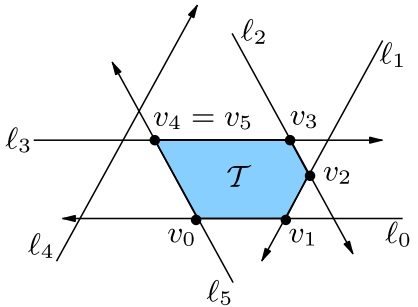

(b) Two wedges containing the same point $x$.

Fig. 4 Construction of $\mathcal{T}$ and illustration of Lemma 2

Proof It suffices to observe that the halfplane to the left of the line is contained in the union of wedges $W_{i}(x)$ and $W_{j}(x)$. (See Fig. 4(b).)

We now show that if two level curves have an intersection in $\mathcal{T}$, then they must have antipodal indices, that is, $i$ and $i+n$. We actually prove the stronger statement that the regions $\mathcal{W}_{i}^{<r}$ do not have any intersection in $\mathcal{T}$, unless they have antipodal indices.

Lemma 3 If $j \neq i$ and $j \neq i+n$, then $\mathcal{W}_{i}^{<r} \cap \mathcal{W}_{j}^{<r} \cap \mathcal{T}=\emptyset$.

Proof Assume by symmetry that $0<(j-i)<n$ and suppose that the two regions intersect at point $x \in \mathcal{T}$. Consider the two wedges $W_{i}(x)$ and $W_{j}(x)$. Since $x$ is contained in $\mathcal{W}_{i}^{<r} \cap \mathcal{W}_{j}^{<r}$, they both contain at most $r+1$ points. By Lemma 2, for all $i^{\prime} \in[i, j-1]$, the oriented line with direction $q_{i^{\prime}} q_{i^{\prime}+1}$ through $x$ has at most $2 r+2$ points of $S$ strictly to its left. This contradicts the fact that $x \in \mathcal{T}$.

We proceed to show that in fact only one pair of level curves can intersect inside $\mathcal{T}$ (a related statement was proved by Pach [11]). This is illustrated in Fig. 5.

Lemma 4 At most one pair of regions $\left\{\mathcal{W}_{i}^{<r}, \mathcal{W}_{i+n}^{<r}\right\}$ intersect in $\mathcal{T}$.

Proof By contradiction, suppose that $y \in \mathcal{W}_{i}^{<r} \cap \mathcal{W}_{i+n}^{<r} \cap \mathcal{T}$ and $z \in \mathcal{W}_{j}^{<r} \cap \mathcal{W}_{j+n}^{<r} \cap \mathcal{T}$ with $j \neq i, i+n$.

First, let us suppose that $z \in W_{i}(y) \cup W_{i+n}(y)$ and focus on the case $z \in W_{i}(y)$. Trivially, $z \in W_{j}(z)$. Thus Lemma 2 implies that for all $i^{\prime} \in[i, j-1]$, the oriented line with direction $q_{i^{\prime}} q_{i^{\prime}+1}$ through $z$ has at most $2 r+2$ points of $S$ strictly to its left, contradicting $z \in \mathcal{T}$. The case $z \in W_{i+n}(y)$ works analogously.

On the other hand, if $z \notin W_{i}(y) \cup W_{i+n}(y)$, then we claim that $y \in W_{j}(z) \cup$ $W_{j+n}(z)$, and a similar argument leads to a contradiction. In order to prove the claim, consider the rays from $y$ parallel to $q_{s} q_{s+1}$ for $s \in[0,2 n-1]$. Then, $z \notin$ $W_{i}(y) \cup W_{i+n}(y)$ implies that $y$ lies (counterclockwise) between either the pair of rays parallel to $q_{i-1} q_{i}$ and to $q_{i} q_{i+1}$ or the pair parallel to $q_{i+n-1} q_{i+n}$ and to 
Fig. 5 Illustration of Lemmas 3 and 4

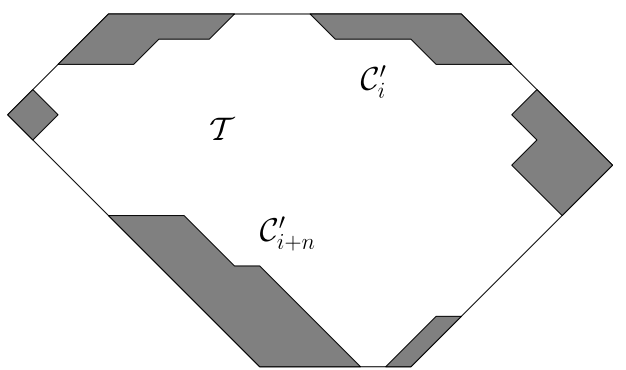

(a) A case where the regions $\mathcal{W}_{i}^{<r} \cap \mathcal{T}$ (in dark) do not intersect.

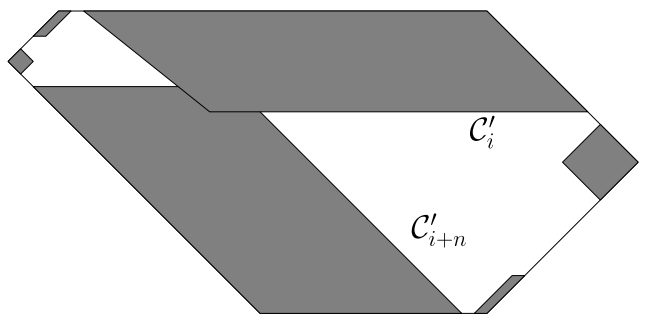

(b) Regions $\mathcal{W}_{i}^{<r}$ and $\mathcal{W}_{i+n}^{<r}$ may intersect in $\mathcal{T}$.

Fig. 6 Proof of Lemma 4

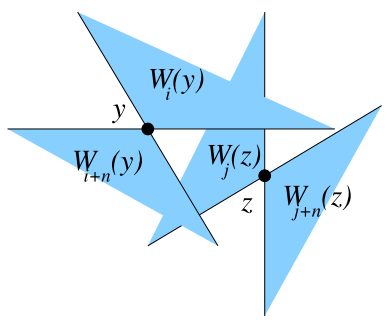

$q_{i+n} q_{i+n+1}$. Given $j \in[i+1, i+n-1]$, we have that $y \in W_{j+n}(z)$ in the first case and $y \in W_{j}(z)$ in the second case (see Fig. 6).

Lemma 5 If $\mathcal{C}_{i}(r)$ intersects the interior of $\mathcal{T}$, then it intersects the boundary of $\mathcal{T}$ at exactly two distinct lines.

We denote the lines by $\ell_{a_{i}}$ and $\ell_{b_{i}}$, so that $\ell_{a_{i}}, v_{i}$, and $\ell_{b_{i}}$ appear in counterclockwise order on the boundary of $\mathcal{T}$, as shown in Fig. 7.

Proof Take any point $x$ on $\mathcal{C}_{i}(r) \cap \mathcal{T}$. We have $v_{i} \in W_{i}(x)$ and $v_{i+n} \in W_{i+n}(x)$, and each of the common supporting lines of those two wedges properly intersects $\mathcal{C}_{i}(r)$ only once. These supporting lines decompose the plane into four wedges: $W_{i}(x)$, $W_{i+n}(x)$, and two additional wedges, each of which contains at least one intersection of $\mathcal{C}_{i}(r)$ with the boundary of $\mathcal{T}$ (see the shaded area in Fig. 7). This implies that we can find two lines $\ell_{a_{i}}$ and $\ell_{b_{i}}$ such that $a_{i} \in[i-n, i-1]$ and $b_{i} \in[i, i+n-1]$, each 
Fig. 7 Proof of Lemma 5

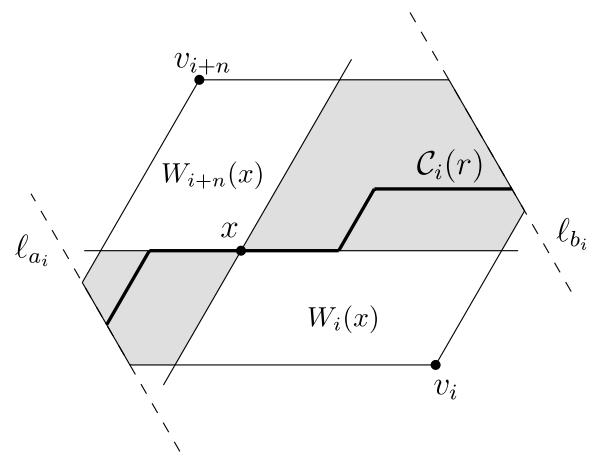

of which contains at least one intersection of $\mathcal{C}_{i}(r)$ with the boundary of $\mathcal{T}$. Since this reasoning is valid for all $x \in \mathcal{C}_{i}(r)$, there are at most two intersections.

Lemma 5 implies that every $\mathcal{C}_{i}(r)$ intersecting $\mathcal{T}$ is such that $i \in\left[a_{i}+1, b_{i}\right]$. Let $\mathcal{C}_{i}^{\prime}(r)$ be the portion of $\mathcal{C}_{i}(r)$ contained in $\mathcal{T}$ :

$$
\mathcal{C}_{i}^{\prime}(r):=\mathcal{C}_{i}(r) \cap \mathcal{T}
$$

Lemma 6 (i) The curve $\mathcal{C}_{i}^{\prime}(r)$ is connected. (ii) If $a_{i} \neq i-1$, then $\mathcal{C}_{j}^{\prime}(r)$ is empty for $j \in\left[a_{i}+1, i-1\right]$. (iii) If $b_{i} \neq i$, then $\mathcal{C}_{j}^{\prime}(r)$ is empty for $j \in\left[i+1, b_{i}\right]$.

Proof Statement (i) follows directly from the fact that $\mathcal{C}_{i}(r)$ is an unbounded curve and intersects $\mathcal{T}$ at most twice (Lemma 5). Statements (ii) and (iii) follow from Lemma 5 and Lemma 3.

Observation 3 If $\mathcal{C}_{i}^{\prime}(r)$ is empty, then any $i$-wedge $W_{i}(x)$ for $x \in \mathcal{T}$ contains at least $r$ points of $S$. In particular, $W_{i}\left(v_{i}\right) \subseteq W_{i}(x)$ and $\left|W_{i}\left(v_{i}\right) \cap S\right| \geq r$.

The combinatorial properties described in this section lay the foundations for the definition of a set of witness wedges in Sect. 4.

\section{Witness Wedges}

We now describe a set of wedges parameterized by a real number $t \in[0,2 n)$ with apex at point $x(t)$ and type $(t)=\lfloor t\rfloor$. We abbreviate $W_{\text {type }(t)}(x(t))=W(t)$. This set of wedges is such that any $i$-wedge containing at least $4 r+5$ points contains a witness wedge $W(t)$. Thus it suffices to color points with respect to those witness wedges.

The wedge $W(t)$ will have its apex on $\mathcal{C}_{i}^{\prime}(r)$ for $t \in[i, i+1)$ if $\mathcal{C}_{i}^{\prime}(r)$ is not empty. More precisely, we let $\sigma_{i}(t), t \in[i, i+1)$ be a parameterization of $\mathcal{C}_{i}^{\prime}(r)$, where $\sigma_{i}(i):=\ell_{a_{i}} \cap \mathcal{C}_{i}^{\prime}(r)$ and $\sigma_{i}(t):=\ell_{b_{i}} \cap \mathcal{C}_{i}^{\prime}(r)$ for $t \in[i+0.9, i+1)$. If $\mathcal{C}_{i}^{\prime}(r)$ is empty, then we distinguish three cases (see Fig. 8):

A. If there is a $j$ such that $i \in\left[a_{j}+1, j-1\right]$, then $\sigma_{i}(t):=\mathcal{C}_{j}^{\prime}(r) \cap \ell_{a_{j}}$ for $t \in$ $[i, i+1)$. 
Fig. 8 Definition of $\sigma_{i}(t)$, when $\mathcal{C}_{i}^{\prime}(r)$ is empty
A.

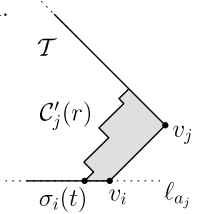

B.

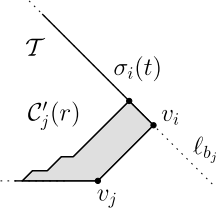

C.

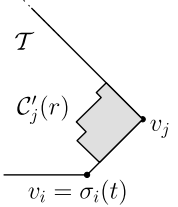

Fig. 9 Illustration of the proof of Lemma 7

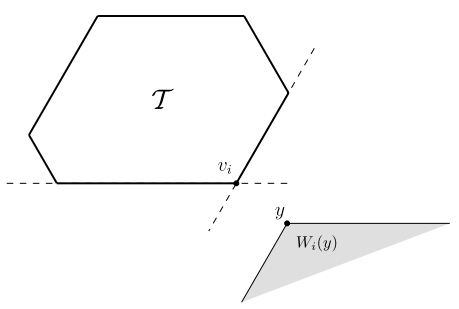

B. If there is a $j$ such that $i \in\left[j+1, b_{j}\right]$, then $\sigma_{i}(t):=\mathcal{C}_{j}^{\prime}(r) \cap \ell_{b_{j}}$ for $t \in[i, i+1)$.

C. Otherwise, $\sigma_{i}(t):=v_{i}$ for $t \in[i, i+1)$.

We define $x(t)$ as the concatenation of the functions $\sigma_{i}(t)$ :

$$
x(t):=\sigma_{\lfloor t\rfloor}(t) .
$$

Lemma 7 For any wedge $W_{i}(y)$ that contains at least $4 r+5$ points of $S$, there is a value $t \in[i, i+1)$ such that $W(t) \subseteq W_{i}(y)$ and $W(t)$ contains at least $r$ points.

Proof Since $W_{i}(y)$ contains at least $4 r+5$ points, it intersects $\mathcal{T}$ : by contradiction, if it does not intersect $\mathcal{T}$, it is completely contained in the union of the two halfplanes adjacent to $\mathcal{T}$ at $v_{i}$ (see Fig. 9). But as each of these halfplanes contains exactly $2 r+2$ points (by definition of $\mathcal{T}$ ), their union can contain at most $4 r+4$ points, and we get the desired contradiction.

Because of that intersection, $W_{i}(y)$ contains a wedge $W_{i}(z)$ such that $z \in \mathcal{T}$.

First suppose that $\mathcal{C}_{i}^{\prime}(r)$ is not empty. Then $\mathcal{C}_{i}^{\prime}(r) \cap W_{i}(y) \neq \emptyset$; otherwise $W_{i}(y)$ cannot contain enough points. If $x(t) \in \mathcal{C}_{i}^{\prime}(r) \cap W_{i}(y)$, then $W(t)$ is contained in $W_{i}(y)$ and contains at least $r$ points.

Now suppose that $\mathcal{C}_{i}^{\prime}(r)$ is empty and refer to cases $\mathrm{A}, \mathrm{B}$, and $\mathrm{C}$ in the preceding. In case $\mathrm{A}$, note that the wedges $W(i)$ and $W(j)$ have the same apex $\mathcal{C}_{j}^{\prime}(r) \cap \ell_{a_{j}}$, $W(j)$ contains at most $r+1$ points, $\ell_{a_{j}}$ has $2 r+3$ points on its left, and $W(i)$ is in the union of $W(j)$ and the halfplane to the left of $\ell_{a_{j}}$ (see Fig. 10). This implies that both $W(j)$ and the halfplane to the left of the oriented line of direction $q_{i} q_{i+1}$ through its apex have at most $3 r+4$ points. Thus $W_{i}(y)$ has its apex outside both $W(j)$ and the halfplane, which implies that $W(i) \subseteq W_{i}(y)$. Because $\mathcal{C}_{i}^{\prime}(r)$ is empty, $|W(i) \cap S| \geq r$. Case B is identical.

In case $\mathrm{C}$, from Observation $3, W_{i}\left(v_{i}\right) \subseteq W_{i}(z)$, and $W_{i}\left(v_{i}\right)$ contains at least $r$ points. Since in that case $x(t)=v_{i}$ for $t \in[i, i+1)$, any value of $t$ in $[i, i+1)$ will work. 
Fig. 10 Case $A$ in the proof of Lemma 7

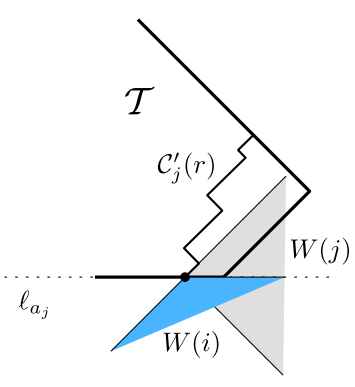

It is natural to view the range $[0,2 n)$ as a counterclockwise parameterization of the points on a unit circle. Thus, in what follows, the real parameter $t$ will be viewed modulo $2 n$, and an interval $\left[t, t^{\prime}\right]$ is the set of points on the circle on a counterclockwise walk from $t$ to $t^{\prime}$.

\section{Reduction to Intervals}

Our goal is to color the points of $S$ with $k$ colors such that any witness wedge $W(t)$ contains at least one point of each color. For each point $p$ in $S$, we consider the set $I(p)$ of witness wedges containing $p$ :

$$
I(p):=\{t \in[0,2 n): p \in W(t)\} .
$$

Intuitively, the following lemma states that if a point $p$ is contained in the wedges for two different values $t$ and $t^{\prime}$ of the parameterization, then $p$ is contained in every wedge $W\left(t^{\prime \prime}\right)$ of the parameterization with $t, t^{\prime \prime}$, and $t^{\prime}$ appearing consecutively in an interval of length less than $n-1$.

Lemma 8 For any point $p \in S$, if $p \in W(t) \cap W\left(t^{\prime}\right)$, where $t^{\prime} \notin[\lfloor t\rfloor, t]$ and type $\left(t^{\prime}\right) \in$ $[$ type $(t)$, type $(t)+n-1]$, then $p \in W\left(t^{\prime \prime}\right)$ for all $t^{\prime \prime} \in\left[t, t^{\prime}\right]$.

Proof There are two cases to consider, depending on type $(t)$ and type $\left(t^{\prime}\right)$ :

- $\operatorname{type}(t)=$ type $\left(t^{\prime}\right)$, or

- type $\left(t^{\prime}\right) \in[$ type $(t)+1$, type $(t)+n-1]$.

In the first case, either $W(t)=W\left(t^{\prime}\right)$, or $x\left(t^{\prime \prime}\right)$ lies on $\mathcal{C}_{i}^{\prime}(r)$ between $x(t)$ and $x\left(t^{\prime}\right)$, by definition of the parameterization. Since $\mathcal{C}_{i}^{\prime}(r)$ is monotone in all directions between $q_{i} q_{i-1}$ and $q_{i} q_{i+1}$, the wedge $W\left(t^{\prime \prime}\right)$ contains the intersection of $W(t)$ and $W\left(t^{\prime}\right)$, and therefore $W\left(t^{\prime \prime}\right)$ contains $p$.

In the second case, without loss of generality, we can assume that type $\left(t^{\prime \prime}\right) \neq$ type $(t)$ and type $\left(t^{\prime \prime}\right) \neq$ type $\left(t^{\prime}\right)$, for otherwise the arguments of the first case apply.

By Lemma 3, $p \notin \mathcal{T}$. Otherwise, it would mean that two wedges $W(t)$ and $W\left(t^{\prime}\right)$ intersect inside $\mathcal{T}$, with type $\left(t^{\prime}\right) \neq$ type $(t)+n$. Also, because $x(t)$ and $x\left(t^{\prime}\right)$ are in $\mathcal{T}$, by the same lemma and reasoning, we get that $x(t) \notin W\left(t^{\prime}\right)$ and $x\left(t^{\prime}\right) \notin W(t)$.

Thus we have the situation depicted in Fig. 11. By the definition of the parameterization, two wedges $W(t)$ and $W\left(t^{\prime}\right)$, with $t^{\prime} \in[t, t+n-1]$ are such that the 


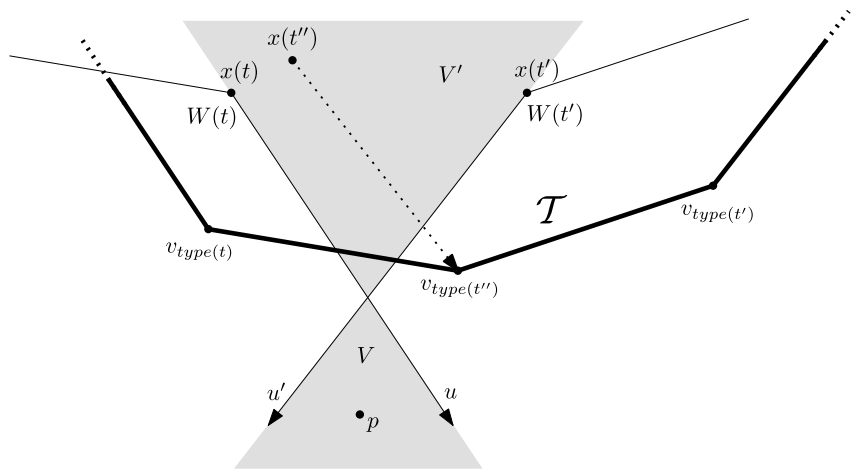

Fig. 11 Second case of Lemma 8. Intersections of the wedges $W(t), W\left(t^{\prime \prime}\right)$ and $W\left(t^{\prime}\right)$

counterclockwise bounding ray $u$ of $W(t)$ intersects the clockwise bounding ray $u^{\prime}$ of $W\left(t^{\prime}\right)$, and the two other rays do not intersect. The point $p$ is in the closed wedge $V$ to the right of $u$ and left of $u^{\prime}$.

Then for any point $q$ in the closed wedge $V^{\prime}$ opposite to $V$, the wedges $W_{j}(q)$ contain $V$ for $j \in\left[\right.$ type $(t)$, type $\left.\left(t^{\prime}\right)\right]$ : indeed, by the definition of the wedges, the absolute angle of the clockwise ray of $W_{j}(q)$ is between that of $W(t)$ and of $W\left(t^{\prime}\right)$, and symmetrically the absolute angle of the counterclockwise ray of $W_{j}(q)$ is between that of $W(t)$ and of $W\left(t^{\prime}\right)$.

To conclude the proof, we will now show that every $x\left(t^{\prime \prime}\right)$ of the parameterization is inside $V^{\prime}$. Recall that, by Lemma $1, W\left(t^{\prime \prime}\right)$ contains $v_{\text {type }\left(t^{\prime \prime}\right)}$, and by definition, the vertices $v_{\text {type }(t)}, v_{\text {type }\left(t^{\prime \prime}\right)}$, and $v_{\text {type }\left(t^{\prime}\right)}$ appear in nonstrictly sequential counterclockwise order on the boundary of $\mathcal{T}$. As $W(t), W\left(t^{\prime \prime}\right)$, and $W\left(t^{\prime}\right)$ cannot intersect strictly inside $\mathcal{T}$, and the ray from $x\left(t^{\prime \prime}\right)$ to $v_{\text {type }\left(t^{\prime \prime}\right)}$ is contained in $W\left(t^{\prime \prime}\right)$, either $x\left(t^{\prime \prime}\right) \in V^{\prime}$ or $x\left(t^{\prime \prime}\right)$ is on the boundary of $\mathcal{T}$. But in that latter case, $x\left(t^{\prime \prime}\right)$ must appear to the left of or on $u$ and to the right of or on $u^{\prime}$ (according to case $\mathrm{A}, \mathrm{B}$, or $\mathrm{C}$ of the definition of the parameterization), therefore it must also be in $V^{\prime}$.

As a consequence, a point corresponds to either an interval of values of the parameterization or a pair of intervals, the corresponding wedges of which are of two types $i$ and $i+n$.

Corollary $1 I(p)$ is either an interval or a pair of intervals $I_{1}(p), I_{2}(p)$ such that type $(t)=i$ for $t \in I_{1}(p)$ and type $(t)=i+n$ for $t \in I_{2}(p)$, where $i$ is such that $\mathcal{W}_{i}^{<r}$ and $\mathcal{W}_{i+n}^{<r}$ intersect in $\mathcal{T}$.

Proof By Lemma 8, $I(p)$ cannot consist of more than two intervals, since otherwise we can find two points $t$ and $t^{\prime}$ satisfying the conditions of Lemma 8 in two distinct intervals.

Now first suppose that no pair $\left\{\mathcal{W}_{i}^{<r}, \mathcal{W}_{i+n}^{<r}\right\}$ intersects in $\mathcal{T}$. Then again the statement is a direct consequence of Lemma 8. Otherwise suppose that $p \in \mathcal{W}_{i}^{<r} \cap \mathcal{W}_{i+n}^{<r}$. Then we must show that $I_{1}(p) \subset[i, i+1)$ and $I_{2}(p) \subset[i+n, i+n+1)$. For contradiction, let $i+1$ be contained strictly in the interior of $I_{1}(p)$. Then there are again 
two points $t \in I_{1}(p) \cap[i+1, i+2)$ and $t^{\prime} \in I_{2}(p) \cap[i+n, i+n+1)$ satisfying the conditions of Lemma 8, a contradiction.

\section{Coloring}

We give an algorithm for coloring the points with $k$ colors so that all wedges $\{W(t)$ : $t \in[0,2 n)\}$ contain all $k$ colors. In the following, we say that a point $p \in S$ covers a point $t \in[0,2 n)$ whenever $t \in I(p)$. We proceed by iteratively removing a covering of $[0,2 n)$, that is, a subset of $S$ the elements of which collectively cover the circle $[0,2 n)$. We use a greedy algorithm to select such a subset; we iteratively expand the cover for $[0, t)$ by selecting a new point that covers the largest interval starting from $t$. Every point in a cover is assigned the same color. By repeating this $k$ times, we ensure that all $k$ colors are represented in each of the wedges $W(t)$ and thus, by Lemma 7, in all wedges containing at least $4 r+5$ points. The key property of the algorithm is that it only requires $r=O(k)$.

A formal description of the algorithm follows. We suppose, without loss of generality, that only the pair $\left\{\mathcal{W}_{0}^{<r}, \mathcal{W}_{n}^{<r}\right\}$ may intersect in $\mathcal{T}$.

\section{Coloring Algorithm}

for $i \leftarrow 1$ to $k$ do:

1. $x \leftarrow 0, S^{\prime} \leftarrow \varnothing$

2. while $\bigcup_{p \in S^{\prime}} I(p) \neq[0,2 n)$ do:

(a) find $p \in S$ such that $y(p):=\max _{t \in[0,2 n)}\{t-x:[x, t] \subseteq I(p)\}$ is maximized

(b) $S^{\prime} \leftarrow S^{\prime} \cup\{p\}$

(c) $x \leftarrow x+y(p)$

3. assign color $i$ to all points in $S^{\prime}$

4. $S \leftarrow S \backslash S^{\prime}$

When every set $I(p)$ is a simple interval, this algorithm greedily colors circular arcs. The following lemma states that in that case, no point on a circle is covered more than a constant number of times per iteration (see Fig. 12).

Fig. 12 Covering the circle $[0,2 n)$ by circular arcs

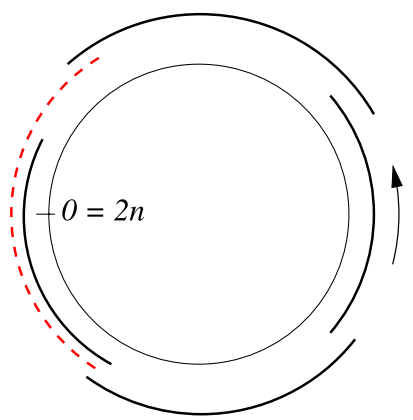


Lemma 9 Suppose that no pair $\left\{\mathcal{W}_{i}^{<r}, \mathcal{W}_{i+n}^{<r}\right\}$ intersects in $\mathcal{T}$ and that there are enough points to perform $j$ iterations of the coloring algorithm. Let $V$ be the set of points colored by the algorithm after the iteration $j$. Then every point of $[0,2 n)$ is covered at most $3 j$ times by points of $V$.

Proof It is sufficient to prove that no point of $[0,2 n)$ is covered more than three times by points of $S^{\prime}$. Note that if no pair of curves intersect, then, by Corollary 1, every set $I(p)$ is an interval. Hence $S^{\prime}$ is a greedy covering of the circle by intervals (i.e., circular arcs).

Let $I(p)$ be the last interval chosen by the algorithm, and consider $S^{\prime \prime}:=S^{\prime}-\{p\}$. Suppose that a point $t$ is covered by more than two points of $S^{\prime \prime}$. Let $a$ and $b$ be the first and the last points chosen, respectively, that cover $t$. The remaining intervals that cover $t$ either extend further than $I(b)$ and should have been chosen instead of $I(b)$, or do not extend further than $I(b)$, in which case $I(b)$ should have been chosen instead. In both cases, we have a contradiction. Hence the points of $S^{\prime \prime}$ do not cover any point of $[0,2 n)$ more than twice. The last interval $I(p)$ can cover some points of the circle a third time. Therefore, every point of $[0,2 n)$ is covered at most three times by points of $S^{\prime}$.

By Corollary 1, a point $p$ may correspond to two intervals on opposite regions of the circle $[0,2 n)$. We show that the following similar property holds.

Lemma 10 Suppose that there are enough points to perform $j$ iterations of the above algorithm, and let $V$ be the set of points colored by the algorithm after the iteration $j$. Then every point of $[0,2 n)$ is covered at most $6 j$ times by points of $V$.

Proof We suppose that $\mathcal{W}_{0}^{<r}$ and $\mathcal{W}_{n}^{<r}$ intersect in $\mathcal{T}$. Otherwise, the statement is implied by Lemma 9. By Lemma 4, only one such pair can intersect. Without loss of generality, we also assume that $\mathcal{C}_{0}^{\prime}$ and $\mathcal{C}_{n}^{\prime}$ are both orthogonal staircases going from top left to bottom right. This setting can always be enforced by symmetry and affine transformation of the points. We assume that $\mathcal{C}_{0}^{\prime}$ is, at some point, above $\mathcal{C}_{n}^{\prime}$, which might cause a point between the two curves to generate one interval on each (see Fig. 13(a)).

We will prove our statement by induction on the number of iterations. Let us show that after the iteration $(j+1)$, no point of $[0,2 n)$ is covered more than $6(j+1)$ times. The induction hypothesis is that this is true for the iterations 0 to $j$, where iteration 0 corresponds to the initial situation. The base case $j=0$ is trivial.

Consider a point $t \in[n, n+1)$, and the corresponding point $x(t)$ on $\mathcal{C}_{n}^{\prime}$. Suppose that this point was covered $\tau$ times in the previous iterations (thus by points of colors 1 to $j$ ). By the induction hypothesis, $\tau \leq 6 j$. We consider the set of points $S^{\prime}$ selected by the algorithm at the iteration $(j+1)$. The sets $I(p)$ start by covering the wedges of type 0 corresponding to points on $\mathcal{C}_{0}^{\prime}$. Let $p$ be the first point of $S^{\prime}$, in order of selection, that also covers $t$. By Corollary $1, p$ only covers two types of wedges, 0 and $n$. Let $q$ be the horizontal projection of $p$ on $\mathcal{C}_{0}^{\prime}$.

Let $p^{\prime}$ be the next point selected by the greedy algorithm. If it covers the point 1 , then by Corollary 1 , it cannot cover any point on $\mathcal{C}_{n}^{\prime}$. Otherwise, since the algorithm 
Fig. 13 Illustration of the proof of Lemma 10

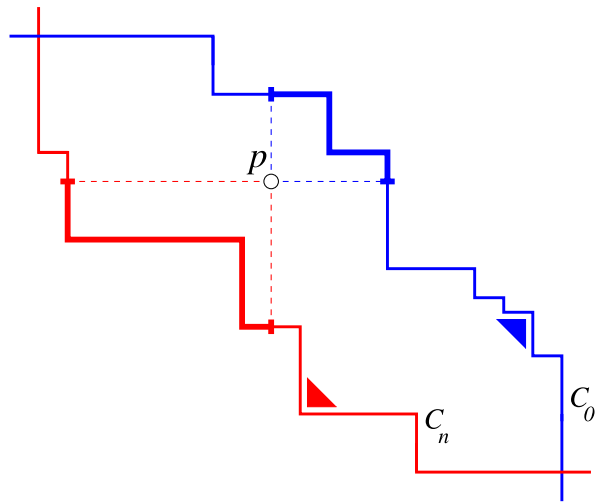

(a) The point $p$ is associated with two intervals $I_{1}(p) \subseteq[0,1)$, and $I_{2}(p) \subseteq[n, n+1)$.

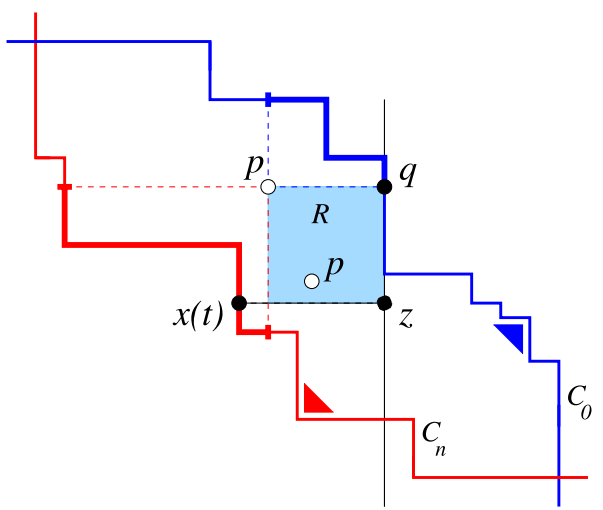

(b) The rectangle $R$.

is greedy, the point is associated with an interval that intersects $I(p)$ and that has the farthest right endpoint. Geometrically, $p^{\prime}$ is the lowest point to the left of the vertical line $\ell$ through $q$. Let $z$ be the projection of $x(t)$ onto $\ell$.

Two cases can occur. First, if $p^{\prime}$ is below $x(t)$, then $t$ is covered at most once, by $p$. On the other hand, if $p^{\prime}$ is above $x(t)$, then $p^{\prime}$ covers $t$.

By the induction hypothesis, $W_{0}(q)$ contains at most $6 j$ colored points. By Observation 1 and since $q \in \mathcal{C}_{0}^{\prime}, W_{0}(q)$ contains at least $r$ points. Hence $W_{0}(q)$ contains at least $r-6 j$ uncolored points (including $p$ and $p^{\prime}$ ). Also, since the algorithm is greedy, $W_{0}(p)$ and $W_{0}(z)$ do not contain uncolored points, otherwise they would have been selected by the algorithm. Hence the orthogonal rectangle $R$ with opposite vertices $p$ and $z$ contains at least $r-6 j$ uncolored points (see Fig. 13(b)).

Since $t$ is covered $\tau$ times and $x(t) \in \mathcal{C}_{n}^{\prime}, W_{n}(x(t))$ can contain at most $r+1-\tau$ uncolored points. The rectangle $R$ is included in $W_{n}(x(t))$, and thus by the previous observation, on $R$, there are at most $(r+1-\tau)-(r-6 j)=6 j+1-\tau$ uncolored points that are both to the right of $\ell$ and above $x(t)$. These, together with $p$ and $p^{\prime}$, are the only points that may cover $t$ after we have covered the interval $[0,1)$. Hence after we have covered the interval $[0,1)$, the points in $[n, n+1)$ cannot be covered 
more than $\tau+(6 j+1-\tau)+2=6 j+3$ times. On the other hand, the points in $[0,1)$ cannot be covered more than $6 j+3$ times.

A similar reasoning holds when the algorithm starts to cover points in the interval $[n, n+1)$. We have to replace $6 j$ by $6 j+3$, since the points on both sides can already be covered $6 j+3$ times. Thus after the $(j+1)$ th iteration, no point is covered more than $6 j+3+3=6(j+1)$ times, which concludes the proof.

Corollary 2 For $r \geq 6 k$, the coloring algorithm finds a $k$-coloring of the points in $S$ such that all wedges $W(t)$ for $t \in[0,2 n)$ contain all $k$ colors.

Note that with this choice of $r$, by the well-known center point theorem, $\mathcal{T}$ is never empty. By Lemma 7, this concludes the proof of Theorem 2, with $\alpha_{Q} k \geq c_{Q} \times(4 \times$ $6 k+5)$ and hence for any $\alpha_{Q} \geq 29 c_{Q}$. By duality, as the polygon $Q$ is symmetric, this implies the following:

Theorem 3 Given a closed centrally symmetric convex polygon $Q$, there exists a constant $\alpha_{Q}$ such that every locally finite $\alpha_{Q} k$-fold covering of the plane by translates of $Q$ can be decomposed into $k$ coverings.

Acknowledgements We thank the anonymous reviewers whose comments helped to greatly improve the presentation of our results.

\section{References}

1. Ali Abam, M., de Berg, M., Poon, S.: Fault-tolerant conflict-free coloring. In: Proceedings of the Canadian Conference on Computational Geometry (CCCG’08) (2008)

2. Abellanas, M., Bose, P., Garcia, J., Hurtado, F., Nicolas, C., Ramos, P.: On properties of higher order Delaunay graphs with applications. Int. J. Comput. Geom. Appl. (to appear)

3. Aloupis, G., Cardinal, J., Collette, S., Langerman, S., Smorodinsky, S.: Coloring geometric range spaces. In: Proceedings of the 8th Latin American Theoretical Informatics (LATIN'08). Lecture Notes in Computer Science, vol. 4957, pp. 146-157. Springer, Berlin (2008)

4. Brass, P., Moser, W.O.J., Pach, J.: Research Problems in Discrete Geometry. Springer, Berlin (2005)

5. Buchsbaum, A., Efrat, A., Jain, S., Venkatasubramanian, S., Yi, K.: Restricted strip covering and the sensor cover problem. In: Proceedings of the ACM-SIAM Symposium on Discrete Algorithms (SODA'07), pp. 1056-1063 (2007)

6. Elekes, M., Matrai, T., Soukup, L.: On splitting infinite-fold covers. Fundam. Math. (to appear)

7. Even, G., Lotker, Z., Ron, D., Smorodinsky, S.: Conflict-free colorings of simple geometric regions with applications to frequency assignment in cellular networks. SIAM J. Comput. 33(1), 94-136 (2004)

8. Gibson, M., Varadarajan, K.: Decomposing coverings and the planar sensor cover problem. In: Proceedings of the 50th Annual IEEE Symposium on Foundations of Computer Science (FOCS09) (2009)

9. Mani, P., Pach, J.: Decomposition problems for multiple coverings with unit balls. Unpublished manuscript (1986)

10. Pach, J.: Decomposition of multiple packing and covering. In: 2. Kolloq. über Diskrete Geom., pp. 169-178. Inst. Math. Univ. Salzburg, Salzburg (1980)

11. Pach, J.: Covering the plane with convex polygons. Discrete Comput. Geom. 1, 73-81 (1986)

12. Pach, J., Tardos, G., Tóth, G.: Indecomposable coverings. In: The China-Japan Joint Conference on Discrete Geometry, Combinatorics and Graph Theory (CJCDGCGT'05). Lecture Notes in Computer Science, vol. 4381, pp. 135-148. Springer, Berlin (2007)

13. Pach, J., Tóth, G.: Decomposition of multiple coverings into many parts. Comput. Geom. Theory Appl. 42(2), 127-133 (2009) 
14. Pálvölgyi, D.: Indecomposable coverings with concave polygons. Discrete Comput. Geom. (to appear)

15. Pálvölgyi, D., Tóth, G.: Convex polygons are cover-decomposable. Discrete Comput. Geom. (to appear)

16. Tardos, G., Tóth, G.: Multiple coverings of the plane with triangles. Discrete Comput. Geom. 38(2), 443-450 (2007) 